\title{
The role of malic enzyme in the regulation of lipid accumulation in filamentous fungi
}

\author{
James P. Wynn, Aidil bin Abdul Hamid $†$ and Colin Ratledge \\ Author for correspondence: Colin Ratledge. Tel: +44 1482 465243. Fax: +44 1482465458. \\ e-mail : c.ratledge $(a$ biosci.hull.ac.uk
}

Lipid Research Group, Department of Biological Sciences, University of Hull, Hull HU6 7RX, UK

\begin{abstract}
The hypothesis is advanced that NADP+-malic enzyme (ME; EC 1.1.1.40) is an important activity in regulating the extent of lipid accumulation in filamentous fungi. In Mucor circinelloides, a fungus capable of accumulating only $\mathbf{2 5 \%}$ (w/w, dry wt) lipid, even under the most propitious conditions, ME disappears 15-20 $\mathrm{h}$ after nitrogen exhaustion, coincident with the cessation of lipid accumulation. In contrast, ME in Mortierella alpina, a fungus capable of accumulating $50 \%$ ( $w / w$, dry wt) lipid, remains active for over $60 \mathrm{~h}$ after $\mathrm{N}$ exhaustion during which time lipid accumulation continues. No other enzyme activity studied, including the lipogenic enzymes acetyl-CoA carboxylase, fatty acid synthase, diacyglycerol acyltransferase, ATP : citrate lyase and the NADPHgenerating enzymes glucose-6-phosphate dehydrogenase, 6-phosphogluconate dehydrogenase and NADP': isocitrate dehydrogenase, demonstrated any correlation with the accumulation of storage lipid in either fungus. Full activity of ME is restored in Mr. circinelloides within $4 \mathrm{~h}$ by adding $\mathrm{NH}_{4}^{+}$to the cultures, but this is prevented by adding cycloheximide as an inhibitor of protein synthesis. This suggests that the decrease in ME activity occurs due to down-regulation of the ME gene.
\end{abstract}

Keywords: lipids, malic enzyme, Mortierella alpina, Mucor circinelloides

\section{INTRODUCTION}

Many eukaryotic micro-organisms (yeast, fungi, algae) accumulate lipid as a storage material when growth becomes limited due to the exhaustion of the nitrogen source, or other nutrient, but where an excess of the carbon source remains (Ratledge, 1997). However, the extent of lipid accumulation differs widely between species, from as little as $3-5 \%(\mathrm{w} / \mathrm{w}$, dry $\mathrm{wt}$ ) to in excess of $80 \%(\mathrm{w} / \mathrm{w}$, dry $\mathrm{wt})$. ATP: citrate lyase (ACL), an enzyme involved in the provision of cytosolic acetylCoA for anabolic processes, including lipogenesis, has been found to be crucial in allowing the accumulation of substantive amounts of lipid $(>20 \%)$ in fungi (Botham \& Ratledge, 1979; Evans \& Ratledge, 1985). However, even in fungi possessing $\mathrm{ACL}$, there exist considerable differences in the capacity to accumulate cell lipid

\footnotetext{
†Present address: Department of Microbiology, University of Kebangsaan Malaysia, 43600 Bangi, Salangor Darul Ehsan, Malaysia.

Abbreviations: ACC, acetyl-COA carboxylase; ACL, ATP: citrate lyase: DAGAT, diacylglycerol acyltransferase; FAS, fatty acid synthase; G-6-PDH, glucose-6-phosphate dehydrogenase; $I C D H$, isocitrate dehydrogenase; $M E$, malic enzyme; 6-PGDH, 6-phosphogluconate dehydrogenase.
}

between closely related species. As strains of yeast that possess ACL but do not accumulate storage lipid have been reported (Ratledge \& Gilbert, 1985) it is evident that the biochemical reason for different species accumulating different amounts of lipid must lie elsewhere than the possession of this activity.

More recently the importance of malic enzyme (ME; EC 1.1.1.40) in the accumulation of storage lipid by fungi has been reported (Wynn \& Ratledge, 1997; Wynn et al., 1997). ME catalyses the reaction

$$
\mathrm{L}-\mathrm{Malate}+\mathrm{NADP}^{+} \rightarrow \text { pyruvate }+\mathrm{CO}_{2}+\mathrm{NADPH}
$$

and is thought to play a key role in the provision of NADPH for both de novo fatty acid biosynthesis and fatty acid desaturation. Inhibitors of ME, such as sesamol, lead to decreased lipid accumulation (Wynn et al., 1997) and mutants of Aspergillus nidulans lacking ME activity also have diminished lipid contents (Wynn \& Ratledge, 1997). This study examines the potential role of ME activity in the regulation of lipid accumulation in two oleaginous fungi-Mucor circinelloides, which has been used commercially to produce an oil rich in $\gamma$-linolenic acid (Ratledge, 1997), which has an upper 


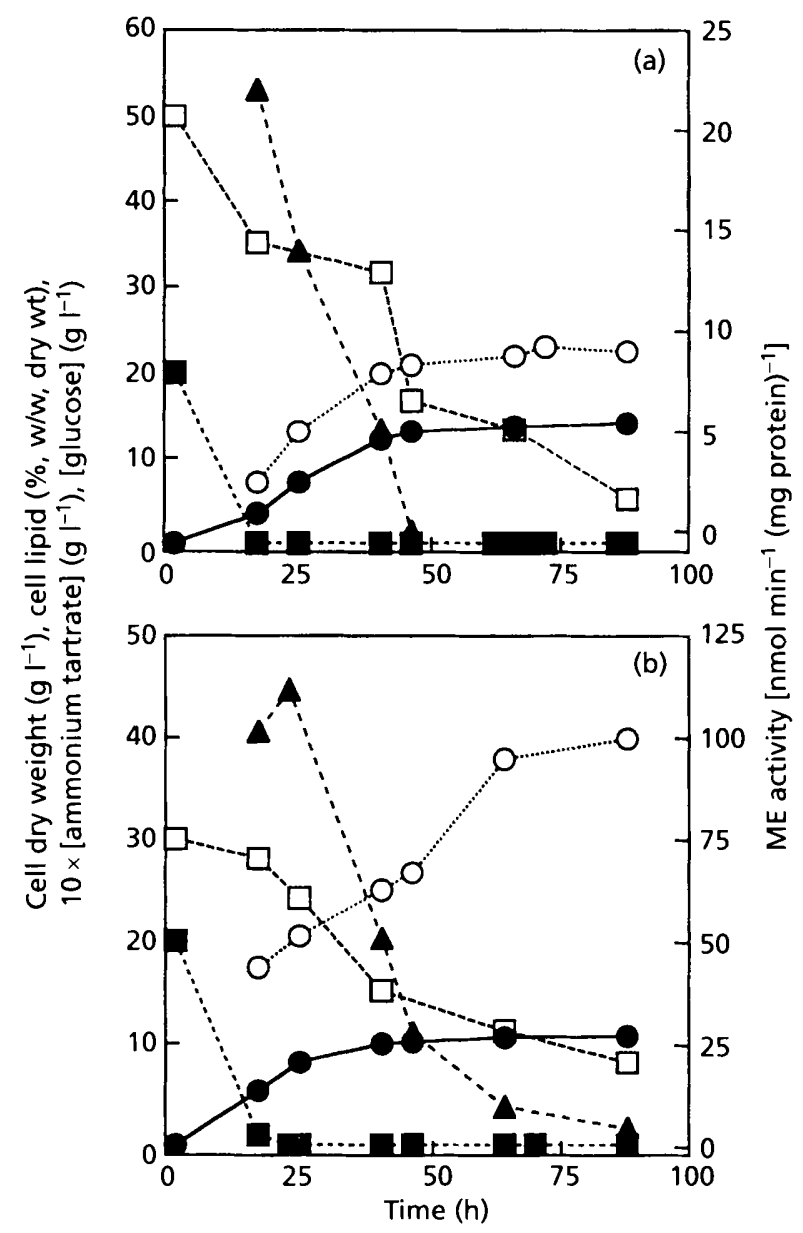

Fig. 1. Growth of, lipid accumulation by and ME activity in $\mathrm{Mr}$. circinelloides (a) and $M t$. alpina (b) grown in a $4 \mathrm{I}$ fermenter on a medium with a $C / N$ ratio of $66: 1$ and $40: 1$, respectively, under batch conditions. 0 , Cell dry weight; $O$, cell lipid; $\mathbf{\square}$ ammonium tartrate concentration; $\square$, glucose concentration; $\Lambda$, ME activity.

limit for lipid accumulation of about $25 \%$ (w/w, dry wt) of the biomass, and Mortierella alpina, which is now being used to produce an oil rich in arachidonic acid, which has an upper limit for lipid accumulation of approximately $50 \%(\mathrm{w} / \mathrm{w}$, dry $\mathrm{wt})$ of the biomass (Shinmen et al., 1989). No previous hypothesis has been advanced to explain why different cells (and this would include plants as well as micro-organisms) have different capacities for storage lipid accumulation.

\section{METHODS}

Chemicals. Unless otherwise stated all fine chemicals were obtained from Sigma.

Cultivation of fungi. Mucor circinelloides CBS 108.16 and Mortierella alpina peyron CBS 696.70 were maintained on Kendrick medium plates (Kendrick \& Ratledge, 1992) and stored at $4{ }^{\circ} \mathrm{C}$. Fungal cultures were initially cultivated in 11 stirred bottles containing $800 \mathrm{ml}$ Kendrick medium. These cultures were incubated for $16 \mathrm{~h}$ at $30^{\circ} \mathrm{C}$ then used to provide a $10 \%(\mathrm{v} / \mathrm{v})$ seed to inoculate $4 \mathrm{l}$ (working volume) fermenters containing modified Kendrick medium, ammonium tartrate $\left(2 \mathrm{~g} \mathrm{l}^{-1}\right)$ and glucose (50 and $30 \mathrm{~g} \mathrm{l}^{-1}$ for $\mathrm{Mr}$. circinelloides and Mt. alpina, respectively). Fermenters were incubated at $30^{\circ} \mathrm{C}$, stirred at 500 r.p.m. with aeration at $0.5 \mathrm{v} / \mathrm{v} \mathrm{min}{ }^{-1}$ and $\mathrm{pH}$ was maintained at 5.5 by automatic addition of $\mathrm{KOH}$ and $\mathrm{HCl}$.

Production of cell extracts. Culture samples were removed periodically from the fermenters for analysis. Biomass was harvested by filtration (under reduced pressure) through a Whatman no. 1 filter and washed with cold purified water. Cell extracts for the determination of enzyme activities other than diacylglycerol acyltransferase (DAGAT) were prepared by suspending mycelia in an extraction buffer containing $100 \mathrm{mM} \mathrm{KH}_{2} \mathrm{PO}_{4} / \mathrm{KOH}$ ( $\mathrm{pH} \mathrm{7.5),20 \%} \mathrm{(w/v)} \mathrm{glycerol,} 1 \mathrm{mM}$ benzamidine/ $\mathrm{HCl}$ and $1 \mathrm{mM}$ dithiothreitol, and disrupted by passage twice through a French pressure cell at $35 \mathrm{MPa}$. Disrupted cell suspensions were centrifuged at $10000 \mathrm{~g}$ for $10 \mathrm{~min}$ and the supernatant retained for enzyme analysis. Extracts for the determination of DAGAT were prepared as described above but the extraction buffer contained $10 \mathrm{mM}$ $\mathrm{KH}_{2} \mathrm{PO}_{4} / \mathrm{KOH}(\mathrm{pH} 7 \cdot 0), 250 \mathrm{mM}$ sucrose and $1 \mathrm{mM}$ EDTA. Protein concentrations were determined using the method of Bradford (1976) with BSA as a standard.

Estimation of enzyme activities. The activities of malic enzyme (ME; EC 1.1.1.40), glucose-6-phosphate dehydrogenase (G-6-PDH; EC 1.1.1.49), 6-phosphogluconate dehydrogenase (6-PGDH; EC 1.1.1.44), $\mathrm{NADP}^{+}$: isocitrate dehydrogenase $\left(\mathrm{NADP}^{+}: \mathrm{ICDH}\right.$; EC 1.1.1.42), fatty acid synthase (FAS; EC 2.3.1.86) and ATP: citrate lyase (ACL; EC 4.1.3.8) were assayed as described by Wynn et al. (1997). Acetyl-CoA carboxylase (ACC; EC 6.4.1.2) was assayed as described by Matthews et al. (1990). Diacylglycerol acyltransferase (DAGAT; EC 2.3.1.20) activity was determined using the assay described by Kamisaka et al. (1993) except that the solvent system used for separating the reaction products was petroleum spirit $\left(40^{\circ}-60^{\circ}\right) /$ diethylether/formic acid $(85: 15: 1$, by vol.) and radioactive spots were quantified using a Bioscan System 400 Imaging TLC plate scanner. All assays were carried out at $30^{\circ} \mathrm{C}$.

Determination of culture dry weight. A $20 \mathrm{ml}$ sample of the culture was harvested onto a washed, dried and weighed filter (Whatman no. 1). The filtrate was retained and analysed for culture glucose and ammonium concentrations (see below). Harvested biomass was washed with distilled water and then dried at $100^{\circ} \mathrm{C}$ to constant weight. The weight of the biomass was determined gravimetrically.

Analysis of culture supernatant. The glucose concentration in the culture medium was determined using a glucose oxidase Perid-Test kit (Boehringer Mannheim), according to the manufacturer's instructions. The ammonium concentration in the culture filtrate was determined using the indophenol test (Chaney \& Marbach, 1962).

Analysis of cell lipid. Fungal biomass was harvested by filtration (Whatman no. 1), washed with distilled water and freeze-dried. Cell lipid was extracted with 2:1 (v/v) chloroform/methanol (Folch et al., 1957) and determined gravimetrically.

Reproducibility of data. All experiments were carried out at least three times, except the experiments to restore ME activity by addition of $\mathrm{NH}_{4}^{+}$which were carried out in duplicate. All data presented are reproducible; however, typical data rather than mean data are presented due to some inter-experiment variation in the time of $\mathrm{NH}_{4}^{+}$exhaustion in the fermenters. 


\section{RESULTS AND DISCUSSION}

Growth and lipid accumulation in Mr. circinelloides and Mt. alpina

Both fungi demonstrated a similar, and typical, growth profile in batch fermentation (Fig. 1). Growth was initially rapid during the nitrogen utilization phase, after which growth slowed, although a small increase in biomass continued, presumably due to the accumulation of storage lipid and hyphal wall thickening.

Lipid accumulated after the exhaustion of the nitrogen source. Initially the rate of lipid accumulation was not dissimilar in the two fungi: at $40 \mathrm{~h}$ both contained $20-25 \%$ (w/w, dry wt) lipid. However, whilst in $M r$.
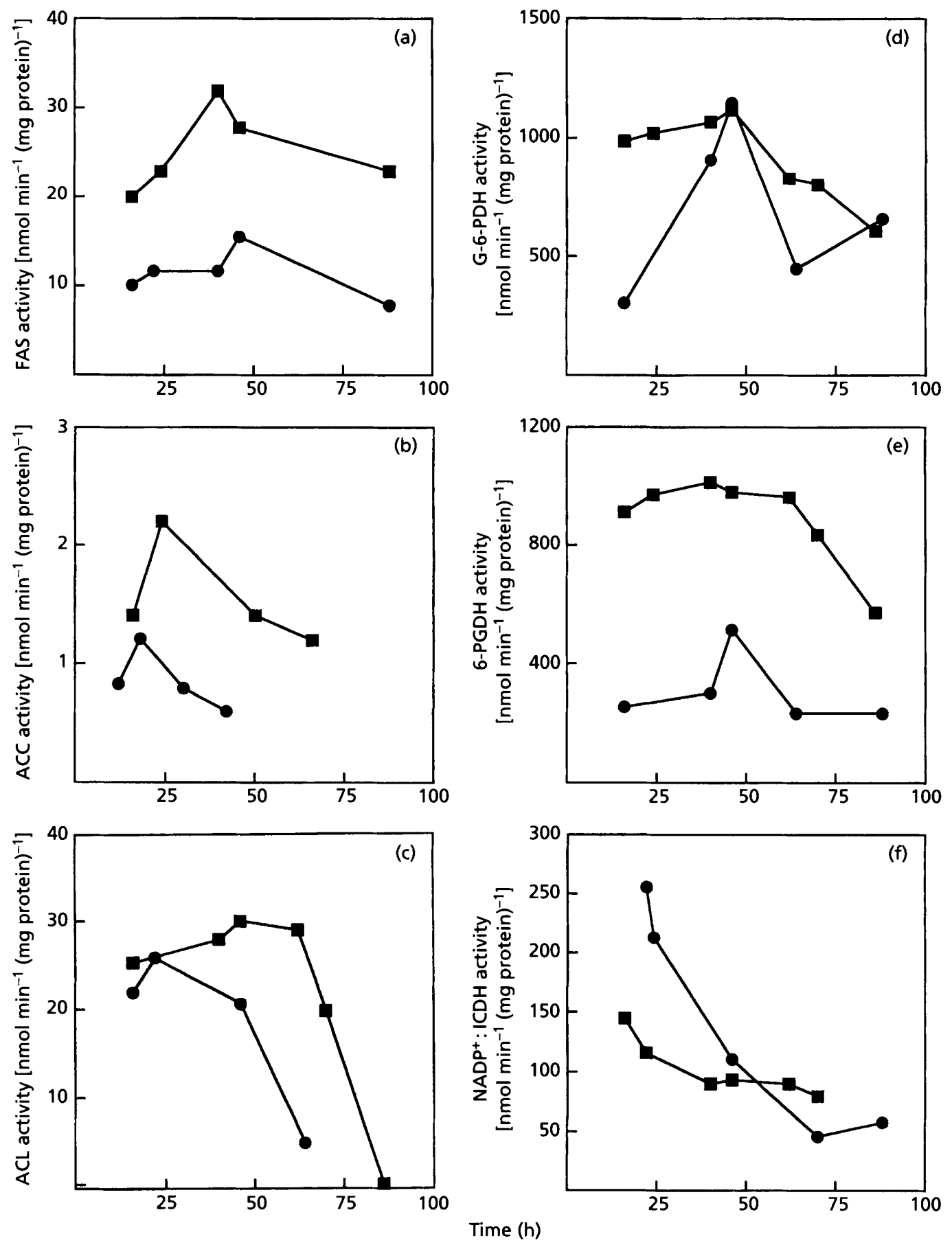

Fig. 2. Activities of key enzymes in Mr. circinelloides $(\boldsymbol{Q})$ and $M t$. alpina $(\boldsymbol{O})$ during growth and lipid accumulation in a fermenter under batch conditions. Enzymes measured were (a) FAS, (b) ACC, (c) ACL, (d) G-6-PDH, (e) 6-PGDH and (f) $\mathrm{NADP}^{+}: \mathrm{ICDH}$. 
Table 1. Activity of DAGAT in Mr. circinelloides and Mt. alpina cultivated in a fermenter, prior to and $24 \mathrm{~h}$ after nitrogen exhaustion from the culture medium

Data are presented as mean values $\pm \operatorname{SEM}(n \geqslant 3)$.

\begin{tabular}{|c|c|c|c|}
\hline & \multirow[t]{2}{*}{ Species } & \multicolumn{2}{|c|}{ DAGAT activity $\left[\mathrm{nmol} \mathrm{min}^{-1}(\mathrm{mg} \text { protein })^{-1}\right]$} \\
\hline & & $\begin{array}{l}\text { Prior to nitrogen exhaustion } \\
\text { in culture medium }\end{array}$ & $\begin{array}{l}24 \mathrm{~h} \text { after nitrogen exhaustion } \\
\text { in culture medium }\end{array}$ \\
\hline \multirow[t]{2}{*}{$-\quad--$} & Mr. circinelloides & $0.29 \pm 0.07$ & $2 \cdot 27 \pm 1 \cdot 62$ \\
\hline & Mt.alpina & $0.50 \pm 0.09$ & $1 \cdot 40 \pm 0 \cdot 35$ \\
\hline
\end{tabular}

circinelloides lipid accumulation ceased after $45 \mathrm{~h}$ (at $25 \%, \mathrm{w} / \mathrm{w}$, dry wt), in Mt. alpina it continued beyond 80 h reaching $40 \%$ (w/w, dry wt).

These results suggest that it is not the rate of lipid synthesis but rather the duration of lipogenesis that determines the extent of lipid accumulation in oleaginous fungi. In this regard, ACC, although probably an enzyme with a regulatory role governing the rate of lipid synthesis in fungi, as has been suggested in plants (Page et al., 1994), is unlikely to be important in determining the extent of lipogenesis. This observation is in accord with those of Kang et al. (1994) and Roesler et al. (1997) in plant systems.

\section{Activities of key enzymes during lipid accumulation in Mr. circinelloides and Mt. alpina}

A range of enzymes involved, or potentially involved, in the biochemistry of oleaginicity were studied in both fungi. These enzymes included those belonging to the lipid biosynthetic pathway itself, ACC, FAS and DAGAT; ACL, which is implicated in the production of cytosolic acetyl-CoA for lipid synthesis in oleaginous yeasts (Botham \& Ratledge, 1979; Evans \& Ratledge, 1985); and a range of enzymes potentially involved in the generation of NADPH for fatty acid synthesis, ME, G-6-PDH, 6-PGDH and NADP ${ }^{+}$:ICDH.

Although the specific activities of the enzymes varied to some degree between the two fungi, the developmental profiles for all but one of the enzymes were similar (Fig. 2), the exception being ME which is discussed below. Activities of the other enzymes studied were detectable many hours after nitrogen exhaustion and, for $M r$. circinelloides, at a time when lipid accumulation had ceased. There was, however, a decline in the activity of some enzymes toward the end of growth, notably ACL and $\mathrm{NADP}^{+}: \mathrm{ICDH}$ (see Fig. 2). FAS (Fig. 2a) though remained active even long after lipid accumulation had ceased in $M r$. circinelloides and had virtually stopped in Mt. alpina. Similarly, DAGAT, which is responsible for the final reaction in the synthesis of the storage triacylglygerols, remained active in both fungi $24 \mathrm{~h}$ after nitrogen exhaustion (see Table 1).

The enzyme activity that demonstrated a different developmental profile between Mr. circinelloides and
Mt. alpina was ME. In Mr. circinelloides the activity of ME was low [20-25 nmol $\left.\min ^{-1}(\mathrm{mg} \text { protein) })^{-1}\right]$ and decreased relatively rapidly after nitrogen exhaustion, becoming undetectable at $45 \mathrm{~h}$ (i.e. $25 \mathrm{~h}$ after $\mathrm{NH}_{4}^{+}$ exhaustion. In contrast, the activity of $\mathrm{ME}$ in $\mathrm{Mt}$. alpina was initially higher $\left[100-110 \mathrm{nmol} \mathrm{min}^{-1}(\mathrm{mg} \text { protein })^{-1}\right]$ and, although its activity decreased after nitrogen exhaustion, it remained active even after $88 \mathrm{~h}$. As equally important as the disappearance of ME activity in $M r$. circinelloides was the timing of its disappearance. In $M r$. circinelloides, ME activity became undetectable at $45 \mathrm{~h}$, coincident with the cessation of lipid accumulation (Fig. 1a). At this time the lipogenic enzymes (ACL, FAS, ACC and DAGAT) and other providers of NADPH (G-6$\mathrm{PDH}, 6-\mathrm{PGDH}$ and $\left.\mathrm{NADP}^{+}: \mathrm{ICDH}\right)$ all remained active (Fig. 2 and Table 1).

In $M r$. circinelloides, therefore, lipid accumulation appears to be limited by a lack of NADPH for lipid synthesis associated with the disappearance of ME. Although our previous work has shown the importance of ME in the provision of NADPH for FAS activity, both in Mr. circinelloides and Aspergillus nidulans (Wynn \& Ratledge, 1997; Wynn et al., 1997), this is the first indication that ME activity may play a crucial role in determining the extent of lipid accumulation in filamentous fungi.

In $M t$. alpina ME activity remained detectable for in excess of $80 \mathrm{~h}$ (Fig. 1b) and this correlated not only with the prolonged period of lipid synthesis in this organism but, as the activity of ME itself began to decline late in the fermentation, with the decline in the rate of lipid accumulation. This slowing in lipid accumulation in $M t$. alpina late in the fermentation when ME activity is in decline suggested that loss of ME activity eventually limited lipid accumulation in this organism too.

The reintroduction of nitrogen $\left(\mathrm{NH}_{4}^{+}\right)$to the medium was used to demonstrate that the disappearance of $\mathrm{ME}$ in $M r$. circinelloides was in response to the exhaustion of the nitrogen source. This resulted in full activity of ME being restored within $4 \mathrm{~h}$ (see Fig. 3). De novo protein synthesis was required for the reinstatement of activity after the addition of $\mathrm{NH}_{4}^{+}$as the addition of cycloheximide $\left(100 \mu \mathrm{g} \mathrm{ml}^{-1}\right.$ ) (a known inhibitor of eukaryotic ribosome function) simultaneously with the introduction of $\mathrm{NH}_{4}^{+}$prevented the recovery of $\mathrm{ME}$ 


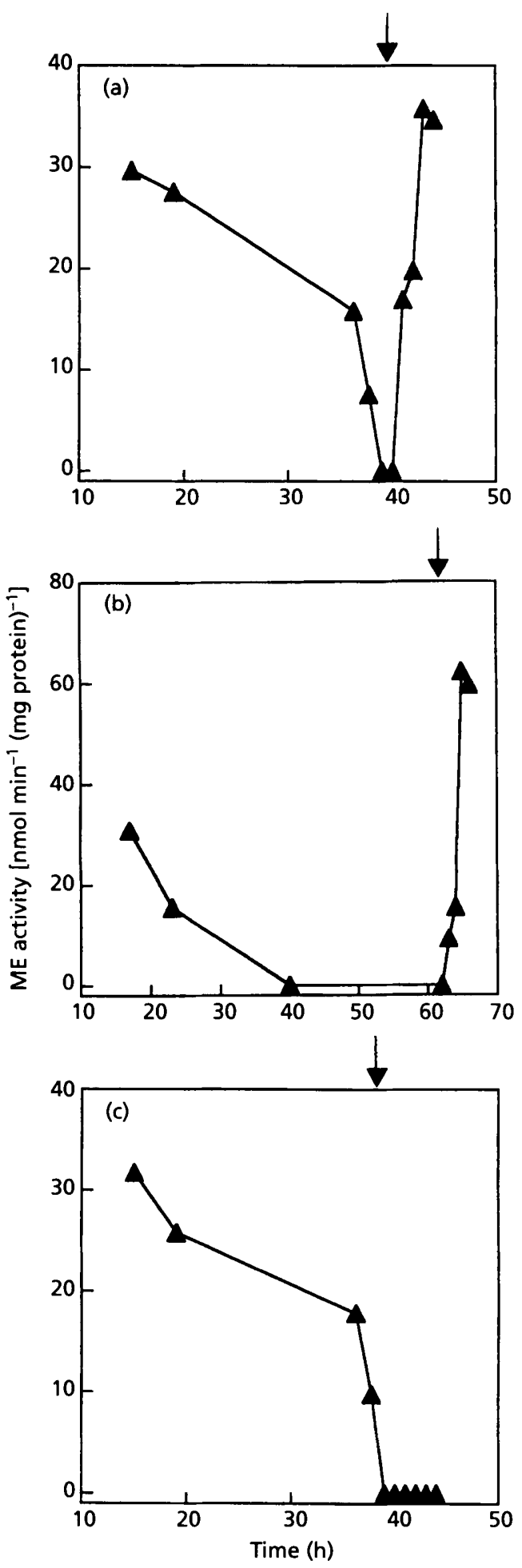

Fig. 3. Reinstatement of ME activity in Mr. circinelloides by the addition of ammonium tartrate (to a final concentration of $2 \mathrm{~g}$ $\left.\mathrm{I}^{-1}\right)$. The reinstatement is possible both immediately after its initial disappearance (a) and $20 \mathrm{~h}$ after the disappearance of activity (b). The addition of cycloheximide at the same time as the reintroduction of nitrogen to the culture prevents the reinstatement of ME activity in Mr. circinelloides (c). The time of addition of ammonium tartrate to the culture is indicated by a down-pointing arrow. activity. It was demonstrated that the effect of cycloheximide was not due to it inhibiting ME by adding cycloheximide to a culture replete with ME activity: under these conditions the activity of ME was not adversely affected. Nor did cycloheximide affect the in vitro assay of ME. Furthermore, the possibility that the disappearance of ME activity after nitrogen exhaustion was the result of the production of an inhibitor was precluded by the addition of a cell-free extract (produced after nitrogen limitation) devoid of ME activity to an extract containing ME activity: no diminution of activity was observed.

\section{DISCUSSION}

Many cells accumulate lipid, from simple eukaryotic yeasts and fungi to seed plants and animal cells (such as adipocytes). The extent to which these cells can accumulate lipid varies enormously with different, but sometimes closely related, species having large differences in their lipogenic capacity. No hypothesis has yet been advanced that can account for these differences though some researchers have suggested that the first committed enzyme of fatty acid synthesis, ACC, could well be the enzyme that controls the rate of lipid synthesis (Page et al., 1994), although even this is a matter of some controversy and doubt (Kang et al., 1994; Roesler et al., 1997). There is, however, no indication that ACC regulates the extent of lipid accumulation in cells. Furthermore, it is clear that the provision of acetyl-CoA itself, as the essential C2 building unit for fatty acid biosynthesis, is not limiting the extent of lipid accumulation. Acetyl-CoA is produced in oleaginous micro-organisms via ACL (Boulton \& Ratledge, 1981a) which could also be the route of acetyl-CoA formation in plants (Ratledge et al., 1997) but, as shown both in this study and in previous work in this laboratory (Boulton \& Ratledge, 1981a, b; Evans \& Ratledge, 1985), ACL activity does not correlate with the extent of lipid accumulation in oleaginous microorganisms. The possibility that DAGAT activity, as the final step in triacylglycerol assembly, plays a key role in the regulation of lipid accumulation has likewise been precluded. In this study, DAGAT activity demonstrated no obvious correlation to the period of active lipid accumulation. This observation is in accord with previous observations in Mortierella ramanniana where DAGAT activity was detectable even after the cessation of lipid accumulation (Kamisaka et al., 1993).

ME appears, at least in the two fungi examined here, to be important in the provision of NADPH for lipid biosynthesis in vivo. None of the other NADPHgenerating enzymes can apparently duplicate this role. Disappearance of ME activity thus appears to cause the cessation of lipid accumulation. No other enzyme activity that we have monitored here, or previously, has shown such a strong correlation with the extent of lipid accumulation.

In light of these findings we suggest that the key factor controlling the extent of lipid accumulation in oleagi- 
nous micro-organisms is not the supply of carbon but the supply of $\mathrm{H}$ equivalents. It has always been assumed that reducing equivalents for biosynthetic purposes could be generated by any NADPH-producing enzyme: G-6-PDH, 6-PGDH, $\mathrm{NADP}^{+}: \mathrm{ICDH}$ or ME. The assumption was that there exists a common pool of NADPH from which any NADPH-requiring enzyme could draw its supply of co-factor, irrespective of the status of the cell. The data obtained in this study, along with our previous published work (Kendrick \& Ratledge, 1992; Wynn et al., 1997; Wynn \& Ratledge, 1997), suggest an important role for ME specifically in the provision of NADPH for lipid synthesis. These observations are not consistent with the existence of a generally available cytosolic 'pool' of NADPH, at least for lipogenesis.

The manner in which ME is integrated into fatty acid biosynthesis remains to be determined. However the 'metabolon' concept advanced by Srere (1987), who showed a physical association between the lipogenic enzymes ACL, ACC and FAS in animal cells, may be one explanation. If a lipogenic metabolon does indeed exist we would suggest that it is likely to include $\mathrm{ME}$ as a provider of NADPH. A physical association between ME and FAS, leading to the channelling of NADPH between the active sites of these two enzymes is, we suggest, the most likely explanation for the apparent importance of ME in the lipogenic process.

The regulation of ME appears to be exerted at a level prior to protein translation as shown by the failure of ME activity to recover in cells treated with cycloheximide. We would suggest that the likely site of regulation of ME activity is at the level of gene expression, although we cannot rule out the possibility that RNA processing plays a role.

Whatever the exact mechanism of regulating ME activity, the identification of this enzyme activity as a potentially important factor in controlling lipid accumulation in cells means that ME may represent an attractive target to those attempting to alter the lipogenic potential of microbial cells. These findings are therefore of importance to those attempting to increase the productivity of micro-organisms for the commercial production of single cell oils in which the extent of lipid accumulation could perhaps be increased by up-regulation of the ME gene. Conversely, in micro-organisms synthesizing other secondary metabolites from acetyl$\mathrm{CoA}$ - such as terpenes and polyketides - the formation of triacylglycerols, which represents a wasteful biosynthetic pathway competing for the carbon supply, could be curtailed by down-regulation of the gene encoding ME.

Furthermore, if the generality of these results can be confirmed for other eukaryotic cells then in plants an increase in seed oil accumulation may also be possible by manipulation of the ME gene. Indeed a key role for $\mathrm{ME}$ in the regulation of plant lipogenesis has already been suggested (Colombo et al., 1997).

\section{ACKNOWLEDGEMENTS}

We would like to thank Mrs Maureen Ewing, Mrs Janet Stephenson and $\mathrm{Mr}$ David Grantham for their expert assistance throughout this work. J. P. Wynn was supported by research grant 21.27.67 from the Biotechnology and Biological Sciences Research Council, UK. A. A. Hamid was supported by a scholarship from the Universiti Kebangsaan, Malaysia.

\section{REFERENCES}

Botham, P. A. \& Ratledge, C. (1979). A biochemical explanation for lipid accumulation in Candida 107 and other oleaginous micro-organisms. J Gen Microbiol 114, 361-375.

Boulton, C. A. \& Ratledge, C. (1981a). Correlation of lipid accumulation in yeasts with possession of ATP:citrate lyase. $J$ Gen Microbiol 127, 169-176.

Boulton, C. A. \& Ratledge, C. (1981b). ATP: citrate lyase - the regulatory enzyme for lipid biosynthesis in Lipomyces starkeyi? J Gen Microbiol 127, 423-426.

Bradford, M. M. (1976). A rapid and sensitive method for the quantitation of microgram quantities of protein utilizing the principle of protein-dye binding. Anal Biochem 72, 248-254.

Chaney, A. L. \& Marbach, E. P. (1962). Modified reagents for the determination of ammonium and urea. Clin Chem 8, 130-132.

Colombo, S. L., Andreo, C. S. \& Podesta, F. E. (1997). Carbon metabolism in germinating Ricinus communis cotyledons. Purification, characterization and developmental profile of NADPdependent malic enzyme. Physiol Plant 101, 821-826.

Evans, C. T. \& Ratledge, C. (1985). Possible regulatory roles of ATP:citrate lyase, malic enzyme and AMP deaminase in lipid accumulation by Rhodosporidium toruloides CBS 14. Can J Microbiol 31, 1000-1005.

Folch, J., Lees, M., Sloane-Stanley, G. H. (1957). A simple method for the isolation of total lipids from animal tissues. J Biol Chem 226, 497-509.

Kamisaka, Y., Yokochi, T., Nakahara, T. \& Suzuki, O. (1993). Characterization of the diacylglycerol acyltransferase activity in the membrane fraction from a fungus. Lipids 28, 583-587.

Kang, F., Ridout, C. J., Morgan, C. L. \& Rawsthorne, S. (1994). The activity of acetyl-CoA carboxylase is not correlated with the rate of lipid synthesis during the development of oilseed rape (Brassicus napus L.) embryos. Planta 193, 320-325.

Kendrick, A. \& Ratledge, C. (1992). Desaturation of polyunsaturated fatty acids in Mucor circinelloides and the involvement of the novel membrane-bound malic enzyme. Eur $]$ Biochem 209, 667-673.

Matthews, J. M., Holtum, J. A. M., Liljegren, D. R., Furness, B. \& Powles, S. B. (1990). Cross-resistance to herbicides in annual ryegrass (Lolium rigidum). 1. Properties of the herbicide target enzymes acetyl-CoA carboxylase and acetolactate synthase. Plant Physiol 94, 1180-1186.

Page, R. A., Okada, S. \& Harwood, J. L. (1994). Acetyl-CoA carboxylase exerts strong flux control over lipid synthesis in plants. Biochim Biophys Acta 1210, 369-372.

Ratledge, C. (1997). Microbial lipids. In Biotechnology, 2nd edn, vol. 7, pp. 133-197. Edited by H. J. Rehm \& G. Reed. Weinheim, Germany: VCH.

Ratledge, C. \& Gilbert, S. C. (1985). Carnitine acetyltransferase activity in oleaginous yeast. FEMS Microbiol Lett 27, 273-275.

Ratledge, C., Bowater, M. D. V. \& Taylor, P. N. (1997). Correlation of ATP: citrate lyase activity with lipid accumulation in developing seeds of Brassica napus L. Lipids 32, 7-12. 
Roesler, K., Shintani, D., Savage, L., Boddupalli, S. \& Ohlrogge, J. (1997). Targetting of the Arabidopsis homomeric acetyl-CoA carboxylase to plastids of rapeseeds. Plant Physiol 113, 75-81.

Shinmen, Y., Shimiza, S., Akimoto, K., Kawashima, H. \& Yamada, H. (1989). Production of arachidonic acid by Mortierella fungi. Appl Microbiol Biotechnol 31, 11-16.

Srere, P. A. (1987). Complexes of sequential metabolic enzymes. Annu Rev Microbiol 56, 89-124.
Wynn, J. P. \& Ratledge, C. (1997). Malic enzyme is a major source of NADPH for lipid accumulation by Aspergillus nidulans. Microbiology 143, 253-257.

Wynn, J. P., Kendrick, A. \& Ratledge, C. (1997). Sesamol as an inhibitor of growth and lipid metabolism in Mucor circinelloides via its action on malic enzyme. Lipids 32, 605-610.

Received 2 February 1999; revised 5 May 1999; accepted 11 May 1999. 